\title{
Rapidly rotating Bose-Einstein condensates in an anharmonic confinement
}

\author{
Li-Hua Lu and You-Quan Li \\ Zhejiang Institute of Modern Physics, Zhejiang University, Hangzhou 310027, P. R. China
}

(Received November 20, 2018)

\begin{abstract}
We examine a rapidly rotating Bose-Einstein condensate in an anharmonic confinement and find that many properties such as the critical rotating frequency and phase diagram are quite different from those in a harmonic trap. We investigate the phase transitions by means of average-vortexapproximation. We find that the vortex lattice consists of a vortex array with a hole in the center of the cloud as the rotating frequency $\Omega$ increases and the vortex becomes invisible when $\Omega$ reaches some value.

PACS numbers: 03.75.Lm, 03.75.Hh
\end{abstract}

a. Introduction: There have been many studies on Bose-Einstein Condensate (BEC) rotating with high angular momentum, of which many features resemble some of the novel solid-state systems such as type-II superconductor and quantum Hall liquid. In a number of earlier experiments [1, 2, 3, 4] where the gas is confined in a harmonic trap, the energetically favored state takes a triangular lattice of singly quantized vortices [5]. In the case of harmonic trapping potential, the centrifugal force would have exactly cancelled the trapping force and the system would have collapsed if $\Omega$ reached to the trap frequency $\omega_{x y}$. Thus the centrifugal force prevents the rotating frequency $\Omega$ from being enhanced over $\omega_{x y}$. This limit makes it impossible for angular momentum to reach the higher magnitude as expected. To resolve this problem, some theoretical studies suggest that $\Omega$ is no longer bounded by $\omega_{x y}$ once an anharmonic term is introduced into the trapping potential. Vortices are successfully created in experiment [6] by adding a quartic term in the trapping potential and their phase transitions are investigate by varying the $\Omega$. The experimental images make the theoretical suggestion that the picture should become richer in an anharmonic trap more conceivable $7,8,19,10,11,12,13]$. Indeed, for $\Omega>\Omega_{c}\left(\Omega_{c}\right.$ is the critical frequency), the gas can only form an array of regular vortices in a harmonic trap. However, in an anharmonic trap, it can also be a state of multiple quantization and a mixed state except for the above state, The mixed state consists of a multiply quantized vortex (hole) at the center of the cloud and singly-quantized vortices around it.

Currently, people's interest extends to the fast rotating BEC which exhibits richer picture. We study the fast rotating BEC in quadratic-plus-quartic trapping potential in this paper. First we determine the critical frequency $\Omega_{c}$ by means of the variational method which differs from that in a harmonic trap. We prove that the system in an anharmonic trap can form multiply-quantized state indeed [14]. Then, we extend the approach proposed by Ho 5] to investigate the phase transitions between the mixed state and the state with an regular vortex lattices. Comparing the energies for different $l$, we obtain the $\Omega_{h}$ over which a hole is generated at the center of the cloud. The increase of $\Omega$ will make the hole to absorb the singly- quantized vortices around it gradually and form a giant vortex state finally. Because the velocity that the hole absorbs the singly-quantized vortices becomes larger and larger, the fragility of the vortex lattice increases. However, recent theoretical study [15] found that much longer time were required for $\Omega \simeq \omega_{x y}$ to reach a well-ordered lattice. Based on the this argument it is reasonable that vortex can not be detected for $\Omega \simeq \omega_{x y}$ in experiment [6].

We consider a Bose-Einstein Condensate confined in an anharmonic trap whose potential is well approximated by a superposition of a quadratic and a quartic potential: 16]

$$
v(\vec{r}) \simeq \frac{1}{2} M \omega_{z}^{2} z^{2}+\frac{1}{2} M \omega_{x y}^{2} r^{2}+\frac{M \omega_{x y}^{2}}{2 a_{\perp}^{2}} \lambda r^{4}, \quad(\lambda>0),
$$

where $M$ is the atomic mass; $\omega_{z}$ and $\omega_{x y}$ are the frequencies of the harmonic potentials along the $z$-axis and in the $x y$ plane $\mathbf{r} \equiv(x, y)$ respectively; $a_{\perp}=\left[\hbar /\left(M \omega_{x y}\right)\right]^{\frac{1}{2}}$ denotes the oscillator length and $\lambda$ a small dimensionless parameter characterizing the strength of the quartic potential which is $\lambda \approx 10^{-3}$ in the experiment [6]. The energy functional is given by

$$
E[\Psi]=\int d^{3} r \Psi^{*}\left(h_{z}+h_{\perp}\right) \Psi+\frac{g}{2} \int d^{3} r|\Psi|^{4},
$$

where $\Psi$ is the condensate wave function, $h_{z}$ and $h_{\perp}$ refer to the single-particle Hamiltonian along $z$-axis and in the $x y$ plane, namely, $h_{z}=-\left(\hbar^{2} / 2 M\right) \nabla_{z}^{2}+\left(M \omega_{z}^{2} z^{2}\right) / 2$ and $h_{\perp}=-\left(\hbar^{2} / 2 M\right) \nabla_{\perp}^{2}+\left(M \omega_{x y}^{2} r^{2}\right) / 2+\left(M \omega_{x y}^{2} \lambda r^{4}\right) /\left(2 a_{\perp}^{2}\right)$.

To find the optical form of $\Psi$ for a rapidly rotating system, we ought to minimize the energy Eq.(2) subject to two constraints. Since both the total number of particles and the total angular momentum are constants, two Lagrange multipliers are introduced such that $\delta\left(E-\mu N-\Omega l_{z}\right)=0$. Here the chemical potential $\mu$ and the rotation frequency $\Omega$ of the trap can guarantee the particle number as well as the angular momentum to be constants [18]. We therefore solve the condensate wave function by minimizing the following GrossPitaevskii functional:

$$
K=\int d^{3} r \Psi^{*}\left[h_{z}+h_{\perp}-\Omega L_{z}-\mu\right] \Psi+\frac{g}{2} \int d^{3} r|\Psi|^{4},
$$


in which $L_{z}$ is the angular momentum along the positive direction of $z$-axis ; $g=\left(4 \pi \hbar^{2} a_{s c}\right) / M$ is the strength of the effective two-body interaction with $a_{s c}$ being the s-wave scatting length.

b. Critical rotational frequency- $\Omega_{c}$ : In reality, for a large condensate, the density profile along $\mathrm{z}$ satisfies the Thomas-Fermi approximation, which is similar to the stationary case. Since the gas rotates around the $z$-axis and its feature in the $x y$ plane is more interesting, we simply assume that the particle density along $\mathrm{z}$ is a constant $\rho$ [16] so that we have a two dimensional quantum system (e.g., $z=0$ plane without loss of generality).

It is instructive to consider the limit case $g=0$ and $\lambda=0$ when the hamiltonian in $x y$ plane is similar to that in the quantum Hall regime. In this case the eigenenergy gives rise to the Landau Levels for which the wave function $\Phi_{m}$ for the lowest Landau Level (LLL) reads: $\Phi_{m} \propto r^{m} \exp (i m \phi) \exp \left(r^{2} / 2 a_{\perp}^{2}\right)$ [17]. In experiment, the $g$ and $\lambda$ are nonzero but their magnitudes are relatively small, thus we can solve the problem by means of the variational method. Let us write the variational wave function as $\Phi=C r^{m} \exp (i m \phi) \exp \left(\beta^{2} r^{2} / 2 a_{\perp}^{2}\right)$ with variational parameter $\beta$. Here $C=\beta^{m+1} /\left[a_{\perp}^{m}\left(\pi a_{\perp}^{2} m !\right)^{1 / 2}\right]$ is the normalization constant. The energy for state $\Psi=\sqrt{\rho} \Phi$ is given by (in units of $\rho \hbar \omega_{x y}$ )

$$
\begin{aligned}
K= & \left.\frac{1}{2}\left(\beta^{2}+\frac{1}{\beta^{2}}\right)-\frac{\Omega}{\omega_{x y}}\right) \Gamma_{1}(m)+\frac{\lambda}{2 \beta^{4}} \Gamma_{2}(m) \\
& +\rho a_{s c} \beta^{2} \Gamma_{3}(m)+\left(\frac{\Omega}{\omega_{x y}}-\mu\right),
\end{aligned}
$$

where $\Gamma_{1}(m)=\beta^{2}\left\langle r^{2}\right\rangle / a_{\perp}^{2}=m+1$ refers to the rms width of the wave function in units of $\beta^{2} / a_{\perp}^{2}, \Gamma_{2}(m)=$ $\beta^{4}\left\langle r^{4}\right\rangle / a_{\perp}^{4}=(m+1)(m+2)$ the expectation value of $r^{4}$, and $\Gamma_{3}(m)=2 \pi a_{\perp}^{2} / \beta^{2} \int d^{2} r|\Phi|^{4}=2 m ! /\left(2^{2 m}(m !)^{2}\right)$ the interaction contribution. Minimizing the energy with respect to the variational parameter $\beta$, we obtain the following two equations with respect to $\beta$ for the cases of $m=0$ (non-vortex configuration) and $m=1$ (one vortex of unit strength), respectively,

$$
\begin{aligned}
\beta_{0}^{6}-\beta_{0}^{2}-4 \lambda+2 \rho a_{s c} \beta_{0}^{6} & =0 \\
2 \beta_{1}^{6}-2 \beta_{1}^{2}-12 \lambda+\rho a_{s c} \beta_{1}^{6} & =0 .
\end{aligned}
$$

Their corresponding energies are

$$
\begin{aligned}
K_{0}= & \left(\frac{1}{2}\left(\beta_{0}^{2}+\frac{1}{\beta_{0}^{2}}\right)-\frac{\Omega}{\omega_{x y}}\right)+\frac{\lambda}{2 \beta_{0}^{4}} \times 2+\rho a_{s c} \beta_{0}^{2} \\
& +\left(\frac{\Omega}{\omega_{x y}}-\mu\right) \\
K_{1}= & \left(\frac{1}{2}\left(\beta_{1}^{2}+\frac{1}{\beta_{1}^{2}}\right)-\frac{\Omega}{\omega_{x y}}\right) \times 2+\frac{\lambda}{2 \beta_{1}^{4}} \times 6+\rho a_{s c} \beta_{1}^{2} \times \frac{1}{2} \\
& +\left(\frac{\Omega}{\omega_{x y}}-\mu\right) .
\end{aligned}
$$

The experiment data $N=3 \times 10^{5}, \omega_{x y}=2 \pi \times 65.6 \mathrm{~Hz}$, $\omega_{z}=2 \pi \times 11.0 \mathrm{~Hz}$, and $a_{s c}=53 \AA$, give rise to $\rho a_{s c} \approx 20$
[16]. Thus we have approximately $\beta_{0}=0.397664$ and $\beta_{1}=0.551786$. For $K_{0}=K_{1}$, we obtain $\Omega_{c} / \omega_{x y}=0.2223$ with $\Omega_{c}$ the critical frequency. The state without vortex is stable when $\Omega<\Omega_{c}$, but when $\Omega>\Omega_{c}$ the state with vortex is stable. For $\lambda=0$, the critical frequency is $\Omega_{c} / \omega_{x y}=0.136$ which is smaller than that for $\lambda \neq 0$. It has been shown [18] that the gas without interaction in a harmonic trap does not generate vortex. The contributions of both interaction and rotating frequency $\Omega$ result in vortex in the condensate. The larger the value $\lambda$ is, the smaller the contribution of the interaction term in the total energy will be. Thus, larger rotating frequency $\Omega$ is required for generating vortex. We examine further state configurations with larger units of vortex, viz., for $m=2,3 \cdots$ [17]. The energy $K_{2}$ is minimized when $\beta_{0}=0.642021$. $K_{1}=K_{2}$ gives $\Omega_{2} / \omega_{x y}=0.7128$. Clearly, when $\Omega_{2} / \omega_{x y}>0.7128$ the $K_{1}$ becomes larger than $K_{2}$, which implies that the state with doubly quantized vortex is more stable than the singly quantized vortex. Unlike the case in harmonic trap where the state with singly quantized vortex (unit quantum number) is merely most favorable 17] when $\Omega>\Omega_{c}$, the stable state in a anharmonic trap favors vortex with large quantum numbers (angular momentum). The faster (the larger $\Omega$ ) the anharmonic trap rotates, the higher the quantum number of the vortex will be.

c. Vortex lattice: We consider the state with a large angular momentum by looking at configurations with a large number of vortices of unit strength centered with a giant vortex of larger strength. Since the dynamics along $z$-axis is identical to the stationary case, the kinetic energy $\left|\nabla_{z} \Psi\right|^{2}$ in $z$ direction is neglectable in ThomasFermi approximation (TFA). Let us write $K=\int d z K(z)$,

$$
\begin{aligned}
K(z)= & \int d^{2} r \Psi^{*}\left[-\frac{\hbar^{2}}{2 m} \nabla_{\perp}^{2}+\frac{1}{2}+\frac{M \omega_{x y}^{2}}{2 a_{\perp}^{2}} \lambda r^{4}-\Omega L_{z}\right. \\
& -\mu(z)] \Psi+\frac{1}{2} f \int d^{2} r|\Psi|^{4},
\end{aligned}
$$

where $\mu(z)=\mu-M \omega_{z}^{2} z^{2} / 2$. We are now in the position to calculate $K(z)$. The condensate wave function is expressed as $\Phi=\sum_{m=0}^{q} C_{m} \Phi_{m} \propto \exp \left(-r^{2} / 2 a_{\perp}^{2}\right) F(u)$ with $F(u)=\prod_{l=1}^{q}\left[u-b_{\alpha}\right]$, the vortex function of $u$. Here $u$ refers to $(x+i y) / a_{\perp}$ and $b_{\alpha}$ the position of vortex [5]. A hole in the center of the gas was shown to be generated with enhancing $\Omega$ in weak repulsive interaction regime 7], which was also notice in numerical simulation [8]. Hence we assume that $\left\{b_{\alpha}\right\}$ form a regular lattice in which the vortex at the center site carries $l$ units of angular momentum while the vortices at the other sites merely carry a unit of angular momentum. Then $F(u)$ can be written as

$$
F(u)=u^{l-1} \prod_{\alpha=1}^{q}\left(u-b_{\alpha}\right),
$$

where $b_{0}=0$ is implied. The general form of $\Psi$ is likely

$$
\Psi=f(z) \Phi, \quad \int d^{2} r|\Phi|^{2}=1,
$$


where $f(z)$ describes the density profile of the gas along $z$-axis and $\Phi=\bar{\Phi} / D(z)$ with

$$
\bar{\Phi}=\exp \left(-r^{2} / 2 a_{\perp}^{2}\right) u^{l-1} \prod_{l=1}^{q}\left(u-b_{\alpha}\right),
$$

and $D^{2}=\int d^{2} r|\bar{\Phi}|^{2}$, the normalization constant for $\Phi$. Now the number constraint $\int d^{2} r|\Psi|^{2}$ becomes $\int d z|f|^{2}=N$. Noting that in the complex coordinate

$$
L_{z}=\hbar\left(u \partial u-u^{*} \partial u^{*}\right),
$$

we can obtain the expectation value of $L_{z}$,

$$
\int \Psi^{*} L_{z} \Psi=\hbar \int\left[\left(r / a_{\perp}\right)^{2}-1\right]|\Psi|^{2} .
$$

Then Eq.(7) becomes,

$$
\begin{aligned}
K(z)= & {\left[-\tilde{\mu}(z)+\frac{\hbar\left(\omega_{x y}-\Omega\right)\left\langle r^{2}\right\rangle_{\Phi}}{a_{\perp}^{2}}\right] f^{2} } \\
& +\frac{M \omega_{x y}^{2}}{2 a_{\perp}^{2}} \lambda f^{2}\left\langle r^{4}\right\rangle_{\Phi}+\frac{1}{2} g I_{\Phi} f^{4}
\end{aligned}
$$

where $\tilde{\mu}(z)=\mu-\hbar \Omega-\left(M \omega_{z}^{2} z^{2} / 2\right),\left\langle r^{2}\right\rangle_{\Phi}=\int d^{2} r r^{2}|\Phi|^{2}$, $\left\langle r^{4}\right\rangle_{\Phi}=\int d^{2} r r^{4}|\Phi|^{2}$ and $I_{\Phi}=\int d^{2} r|\Phi|^{4}$. The minimization of the energy is performed by the variation of the parameter $\left\{b_{\alpha}\right\}$.

To evaluate $\left\langle r^{2}\right\rangle_{\Phi},\left\langle r^{4}\right\rangle_{\Phi}$ and $I_{\Phi}$, we note that,

$$
|\bar{\Phi}|^{2}=e^{-H}, \quad H=H_{1}+H_{2},
$$

where $H_{1} \equiv r^{2} / a_{\perp}^{2}-2 \sum_{\alpha} \ln \left|\vec{r}-\overrightarrow{b_{\alpha}}\right|$ and $H_{2} \equiv$ $-2(l-1) \ln |\vec{r}|$. By means of the Fourier transform, one obtains 5],

$$
\begin{aligned}
\nabla_{\perp}^{2} H_{1} & =\frac{1}{\sigma^{2}}-\frac{4 \pi}{v} \sum_{k \neq 0} \cos \vec{k} \cdot \vec{r}, \\
|\bar{\Phi}|^{2} & =r^{2(l-1)} e^{-\left(r^{2} / \sigma^{2}\right)} \prod_{k \neq 0} \exp \left(\zeta_{k} \cos \vec{k} \cdot \vec{r}\right), \\
\frac{1}{\sigma^{2}} & =\frac{1}{a_{\perp}^{2}}-\frac{\pi}{v}, \quad \zeta_{k}=\frac{4 \pi}{v|\vec{k}|} .
\end{aligned}
$$

In the average-vortex approximation [5], the $|\bar{\Phi}|^{2}$ becomes $|\bar{\Phi}|^{2}=r^{2(l-1)} \exp -\left(r^{2} / \sigma^{2}\right)=h(r) \exp -\left(r^{2} / \sigma^{2}\right)$; here $h(r) \equiv r^{2(l-1)}$ is a slowly varying envelope function, which gives rise to the components in higher Landau Levels [21]; the $\Phi$ then becomes $|\bar{\Phi}|^{2}=$ $\left(r^{2(l-1)} \exp \left(-r^{2} / \sigma^{2}\right)\right) /\left(\pi \Gamma(l) \sigma^{2 l}\right)$. Using the above result, we can easily evaluate the values. $\left\langle r^{2}\right\rangle_{\Phi}=$ $l \sigma^{2},\left\langle r^{4}\right\rangle_{\Phi}=l(l+1) \sigma^{4}$, and $I_{\Phi}=\xi /\left(2 \pi \sigma^{2}\right), \quad \xi \equiv$ $(2 l-1) ! /\left(2^{2 l-2}[(l-1) !]^{2}\right)$. Substituting these results into $\mathrm{Eq}(12)$, we have

$$
\begin{aligned}
& K(z)=\left[-\tilde{\mu}(z)+\frac{l \hbar\left(\omega_{x y}-\Omega\right) \sigma^{2}}{a_{\perp}^{2}}\right] f^{2} \\
& +\frac{\hbar \omega_{x y} \lambda}{2 a_{\perp}^{4}} l(l+1) \sigma^{4} f^{2}+\frac{\hbar \omega_{x y} a_{s c} a_{\perp}^{2}}{\sigma^{2}} f^{4} \xi .
\end{aligned}
$$

Minimizing the energy, we obtain two equations,

$$
\begin{aligned}
\frac{\partial K(z)}{\partial f}= & 2\left[-\tilde{\mu}(z)+\frac{l \hbar\left(\omega_{x y}-\Omega\right) \sigma^{2}}{a_{\perp}}\right] f+4 \frac{\hbar \omega_{x y} a_{s c} a_{\perp}^{2}}{\sigma^{2}} \xi f^{3} \\
& +\frac{\hbar \omega_{x y} \lambda}{a_{\perp}^{4}} l(l+1) \sigma^{4} f=0 \\
\frac{\partial K(z)}{\partial \sigma}= & \frac{2 l \hbar\left(\omega_{x y}-\Omega\right) \sigma}{a_{\perp}^{2}} f^{2}+\frac{2 \hbar \omega_{x y} \lambda}{a_{\perp}^{4}} l(l+1) \sigma^{3} f^{2} \\
& -\frac{2 \hbar \omega_{x y} a_{s c} a_{\perp}^{2}}{\sigma^{3}} f^{4} \xi=0 .
\end{aligned}
$$

In the light that the above nonlinear equations are difficult to solve analytically, we study those equations for the cases of $\lambda=0$ and $\lambda \neq 0$ so as to capture some physical implications.

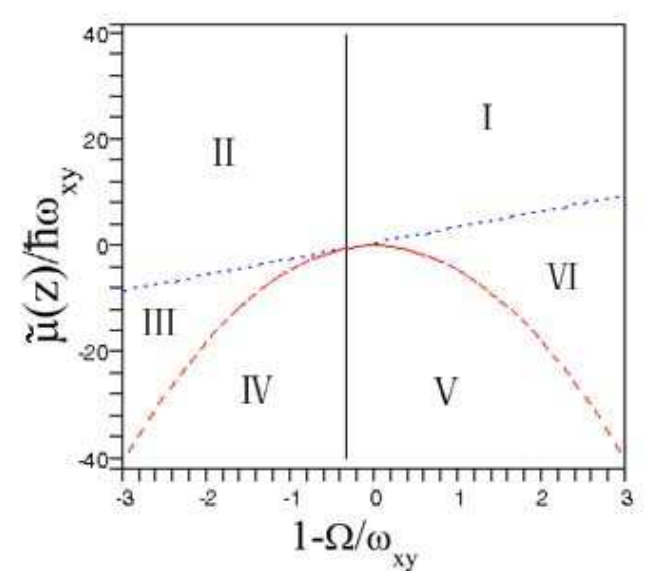

FIG. 1: (color on line) The phase diagram in the plane of $\tilde{\mu}(z)$ versus $\left(1-\Omega / \omega_{x y}\right)$ which is divided by the parabolic curve $\tilde{\mu}(z)=-9 \hbar \omega_{x y} l\left(1-\Omega / \omega_{x y}\right)^{2} / 10(l+1) \lambda$, the oblique line $\tilde{\mu}(z)=\hbar \omega_{x y}\left[2.5 l(l+1) \lambda+3 l\left(1-\Omega / \omega_{x y}\right)\right]$ and the vertical line $\tilde{\mu}(z)=-5 \hbar \omega_{x y}(l+1) \lambda / 3$.

For $\lambda=0$, viz., the gas is confined in a harmonic trap,

$$
\begin{aligned}
\left(\frac{\sigma^{2}}{a_{\perp}^{2}}\right)_{0} & =\frac{\tilde{\mu}(z)^{2}}{3 l \hbar\left(\omega_{x y}-\Omega\right)}, \\
\left(a_{s c} f^{2}\right)_{0} & =\frac{[\tilde{\mu}(z)]^{2}}{9 l^{2} \hbar^{2} \omega_{x y}\left(\omega_{x y}-\Omega\right) \xi} .
\end{aligned}
$$

Substituting Eq.(17) into Eq.(15), we obtain,

$$
[K(z)]_{0}=\frac{[\tilde{\mu}(z)]^{3}}{27 \hbar^{2} \omega_{x y}\left(\omega_{x y}-\Omega\right) a_{s c}}\left(-\frac{2}{l^{2} \xi}+\frac{1}{l^{3} \xi}\right) \propto \alpha
$$

in which $\alpha \equiv\left(-\frac{1}{l^{2} \xi}+\frac{1}{l^{3} \xi}\right)$ [19], for example, $[\alpha]_{l=1}=-1$, $[\alpha]_{l=2}=-3 / 4,[\alpha]_{l=3}=-40 / 81$ etc. This implies that the energy of the system becomes larger with increase of $l$. Thus the hole in the center of the system can not be formed in a harmonic trap, which also be confirmed by variational wave function $\Phi=$ 

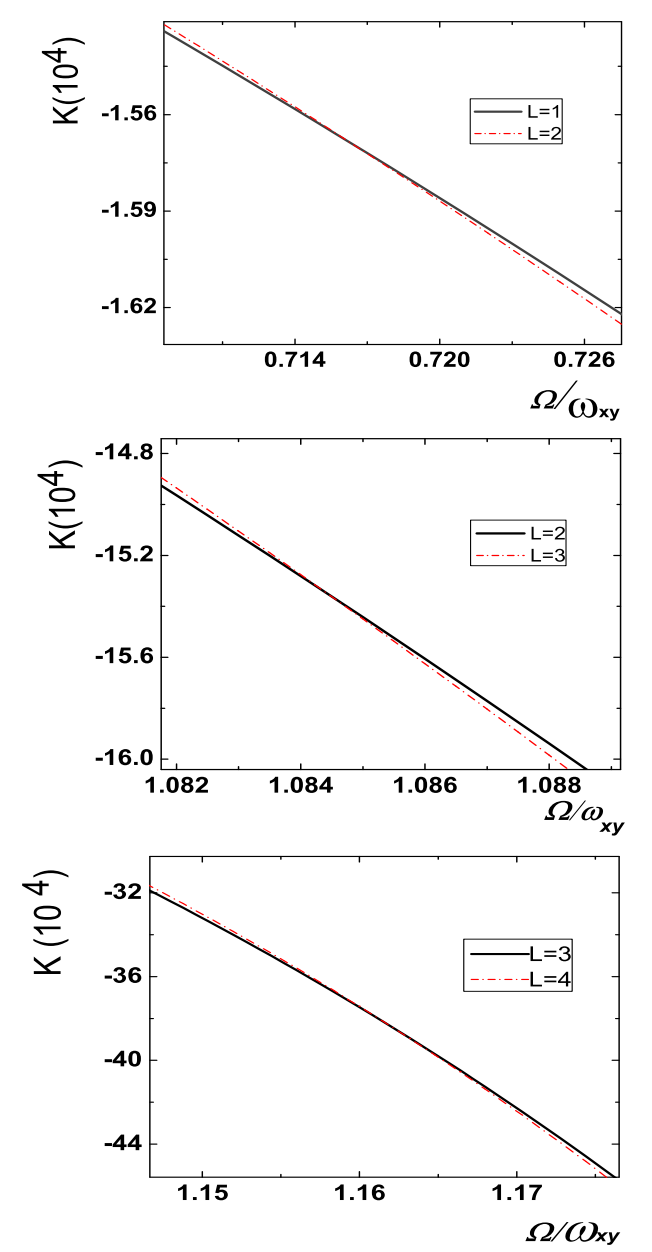

FIG. 2: (color on line) The curves of $K$ versus $\Omega / \omega_{x y}$ in unit of $\left(2 \hbar \omega_{x y}\right)^{3 / 2} M^{-1 / 2} /\left(2 \omega_{z} a_{s c}\right)$ for different $l$ with parameters $N=3 \times 10^{5}, \omega_{x y}=2 \pi \times 65.6, \omega_{z}=2 \pi \times 11.0, a_{s c}=53 \AA$.

$C r^{m} \exp (i m \phi) \exp \left(\beta^{2} r^{2} / 2 a_{\perp}^{2}\right)$ by regarding $\beta$ as variation parameter.

For $\lambda \neq 0$, viz., the gas is confined in an anharmonic trap,

$$
\begin{aligned}
\left(\frac{\sigma^{2}}{a_{\perp}^{2}}\right)_{ \pm} & =-\frac{3\left(1-\Omega / \omega_{x y}\right)}{5(l+1) \lambda} \\
& \pm \frac{\sqrt{9\left(1-\Omega / \omega_{x y}\right)^{2}+[10(l+1) \lambda \tilde{\mu}(z)] /\left(l \hbar \omega_{x y}\right)}}{5(l+1) \lambda} \\
\xi a_{s c} f^{2} & =\frac{l\left(1-\Omega / \omega_{x y}\right) \sigma^{4}}{a_{\perp}^{4}}+\frac{\lambda l(l+1) \sigma^{6}}{a_{\perp}^{6}} .
\end{aligned}
$$

Since $\sigma \geq a_{\perp}$, the condition for the existence of solution of the above equations is plotted in Fig [1 In the regime I and II, only the root of $\left(\sigma^{2} / a_{\perp}^{2}\right)+$ satisfies the condition $\sigma^{2} \geq a_{\perp}^{2}$. In the regime III, both $\left(\sigma^{2} / a_{\perp}^{2}\right)_{ \pm}$satisfy that condition, which implies the system has double stable states and two possible configurations of vortex lattice can be formed. The vortices in this regime become invisible for it is easy to vary between those two configurations due to fluctuation. In the other regimes, $\left(\sigma^{2} / a_{\perp}^{2}\right)_{ \pm}$do not satisfy that condition. In ThomasFermi approximation, $\mu=M R_{i}^{2} \omega_{i}^{2} / 2, R_{i}$ stand for the maximum extents of condensate and $\omega_{i}$ for the frequencies in three direction 18]. According to the experimental data [1, 2], the strait line $\tilde{\mu}(z)=\mu-\hbar \Omega-M \omega_{z}^{2} z^{2} / 2$ must cross the regimes I, II and VI but not cross the other regimes. Thus the condition $\sigma^{2} \geq a_{\perp}^{2}$ becomes $\tilde{\mu}(z) \geq \hbar \omega_{x y}\left[2.5 l(l+1) \lambda+3 l\left(1-\Omega / \omega_{x y}\right)\right]$. Note that for the same rotating frequency $\Omega$ with the growing of $l$, the maximum extent of condensate along z-axis $R_{z}$ will decrease,

$$
R_{z}^{2} \propto \mu / \hbar \omega_{x y}-\Omega / \omega_{x y}-2.5 l(l+1) \lambda-3 l\left(1-\Omega / \omega_{x y}\right) .
$$

For simplicity, we assume straight vortex lines [7] so that the (2D) density of vortex remains a constant along $z$ axis, then we obtain $K=2 R_{z} K(0)$.

We plot the values of $K$ versus $\Omega / \omega_{x y}$ for different $l$ in Fig 2 We can see that with increasing $\Omega$ the state becomes a mixed phase with a multiply quantized vortex (hole) at the center of the cloud and singly-quantized vortices around it [14]. This assures that a fast rotating Bose-Einstein condensate confined in a quadratic-plusquartic potential can generate a hole in the center of the gas which is in agreement with the theoretical and experimental studies 6, 8]. Our calculation shows that if $\Omega$ increases slowly, an array of vortices is formed at first (just like in harmonic trap); then a hole carrying two unit of angular momentums is generated when $\Omega_{h} / \omega_{x y} \approx 0.716$, which keeps until it reaches $\Omega / \omega_{x y} \approx 1.084$. With the increasing of $\Omega$ the hole will absorb the singly-quantized vortices around it whose angular momentum becomes larger and larger. Looking at the positions of intersections in the three graphs in Fig[2 it is easy to find that the velocity of absorbing the singly-quantized vortices becomes larger and larger, and finally a giant vortex carrying all the angular momentums will be formed. When $\Omega / \omega_{x y}>1.084$, every configurations of the vortex lattice remains for a short time, so it is difficult to detect the vortex. Our result can also explain why no vortex lines are detectable in the regime when $\Omega$ reaches to some large value [6].

d. Conclusion: Employing the approach of Ref. [5], we obtained the new form of the condensate wave function which is essentially the Lowest Landau Level (LLL) wave function with a regular lattice of vortices multiplied by a slowly varying envelope function. Using the variational trial function, we obtained the critical value $\Omega_{h}$ and proved that the fragility of the vortex lattice could be enhanced by increasing $\Omega$. This result is in agreement with the experiment result [20] where the gas was trapped in a harmonic potential. In the experiment [20], the vortex lattice remains ordered, but its elastic shear strength is drastically reduced by increasing $\Omega$. This implies that even a minor perturbation to the cloud can cause the lat- 
tice to melt. In the regime with very small $\lambda$, the quartic term also provide a perturbation to destroy the vortex lattice, it is therefore invisible when the $\Omega$ reaches some large value [6].

The work is supported by NSFC Grant No.10225419.
[1] E. Hodby, G. Hechenblaikner, S. A. Hopkins, O. M. Maragò and C.J.Foot, Phys. Rev. Lett. 88, 010405 (2002).

[2] P. C. Haljan, I. Coddington, P. Engels, and E. A. Cornell $^{*}$, Phys. Rev. Lett. 87, 210403 (2001).

[3] K. W. Madison, F. Chevy, W. Wohlleben, and J. Dalibard, Phys. Rev. Lett. 84, 806 (2000).

[4] J. R. Abo-Shaeer, C.Raman, J. M. Vogels, and W. Ketterle, Science 292, 476 (2001).

[5] Tin-Lun Ho, Phys. Rev. Lett. 87, 060403 (2001).

[6] Vincent Bretin, Sabine Stock, Yannick Seurin, and Jean Dalibard, Phys. Rev. Lett. 92, 050403 (2004).

[7] UweR. Fischer and Gordon Baym, Phys. Rev. Lett. 90, 140402 (2003).

[8] Kenichi Kasamatsu, Makoto Tsubota, and Masahito Ueda, Phys. Rev. A 66, 053606 (2002).

[9] E. Lundh, Phys. Rev. A 65, 043604 (2002).

[10] A. L. Fetter, Phys. Rev. A 64, 063608 (2001).

[11] E. Lundh, A. Collin and K.-A. Suominen, Phys. Rev. Lett. 92,070401 (2004).

[12] T. P. Simula, A. A. Penckwitt and R. J. Ballagh, Phys.
Rev. Lett. 92, 060401 (2004).

[13] A. Aftalion and I. Danaila, Phys. Rev. A 69,033608 (2004).

[14] A. D. Jackson, G. M. Kavoulakis and E. Lundh, Phys. Rev. A 69, 053619 (2004).

[15] A. Aftalion and I. Danaila, cond-mat/0309668

[16] A. D. Jackson and G. M. Kavoulakis, Phys. Rev. A 70, 023601 (2004).

[17] V. Subrahmanyam, Phys. Rev. A 67, 035601 (2003).

[18] C. J. Pethick and H. Smith, Bose-Einstein Condensation in Dilute Gass, Chap. 6,9 (Cambridge University press 2002).

[19] Erich J. Mueller* and Tin-Lun HO, Phys. Rev. Lett. 88, 180403 (2002).

[20] V. Schweikhard, I. Coddington, P. Engels, V. P. Mogendorff and E. A. Cornell, Phys. Rev. Lett 92, 040404 (2004).

[21] Gentaro Watanabe, Gordon Baym, and C. J. Pethick, Phys. Rev. Lett 93, 190401 (2004). 